\title{
A mutant Escherichia coli that attaches peptidoglycan to lipopolysaccharide and displays cell wall on its surface
}

\author{
Marcin Grabowicz¹, Dorothee Andres², Matthew D Lebar², Goran Malojčić ${ }^{2 \dagger}$, \\ Daniel Kahne ${ }^{2,3 *}$, Thomas J Silhavy ${ }^{1 *}$
}

1Department of Molecular Biology, Princeton University, Princeton, United States; 2Department of Chemistry and Chemical Biology, Harvard University, Cambridge, United States; ${ }^{3}$ Department of Biological Chemistry and Molecular Pharmacology, Harvard Medical School, Boston, United States

\begin{abstract}
*For correspondence: kahne@ chemistry.harvard.edu (DK); tsilhavy@princeton.edu (TJS)
\end{abstract}

Present address: †Syros Pharmaceuticals Inc., Watertown, USA

Competing interests: The authors declare that no competing interests exist.

Funding: See page 9

Received: 29 October 2014 Accepted: 24 December 2014 Published: 31 December 2014

Reviewing editor: Eduardo $\mathrm{A}$ Groisman, Yale University/HHMI, United States

(c) Copyright Grabowicz et al. This article is distributed under the terms of the Creative Commons Attribution License, which permits unrestricted use and redistribution provided that the original author and source are credited.
Abstract The lipopolysaccharide (LPS) forms the surface-exposed leaflet of the outer membrane (OM) of Gram-negative bacteria, an organelle that shields the underlying peptidoglycan (PG) cell wall. Both LPS and PG are essential cell envelope components that are synthesized independently and assembled by dedicated transenvelope multiprotein complexes. We have identified a point-mutation in the gene for O-antigen ligase (WaaL) in Escherichia coli that causes LPS to be modified with PG subunits, intersecting these two pathways. Synthesis of the PG-modified LPS (LPS*) requires ready access to the small PG precursor pool but does not weaken cell wall integrity, challenging models of precursor sequestration at PG assembly machinery. LPS* is efficiently transported to the cell surface without impairing OM function. Because LPS* contains the canonical vancomycin binding site, these surface-exposed molecules confer increased vancomycin-resistance by functioning as molecular decoys that titrate the antibiotic away from its intracellular target. This unexpected LPS glycosylation fuses two potent pathogen-associated molecular patterns (PAMPs). DOI: 10.7554/eLife.05334.001

\section{Main text}

A peptidoglycan (PG) cell wall is an essential extracytoplasmic feature of most bacteria (Singer et al., 1989); this essentiality has made its biogenesis a fruitful target for antibiotics, including vancomycin and penicillin. The cell wall is directly exposed to the extracellular milieu in Gram-positive bacteria, but is shielded in Escherichia coli and other Gram-negative species by a highly selective permeability barrier formed by the outer membrane (OM). The OM prevents influx of antibiotics, such as vancomycin, restricting their access to intracellular targets (Eggert et al., 2001; Ruiz et al., 2005). Lipopolysaccharide (LPS) forms the surface-exposed outer leaflet of the OM and is key to the barrier function (Osborn et al., 1972; Kamio and Nikaido, 1976; Nikaido, 2003). LPS is a glycolipid consisting of a 'lipid A' anchor within the bilayer, and a set of covalently attached distal 'core' saccharides (Raetz and Whitfield, 2002). LPS is made at the cytosolic leaflet of the inner membrane (IM) before being flipped to the periplasmic leaflet (Zhou et al., 1998). A transenvelope complex of seven lipopolysaccharide transport proteins (LptABCDEFG) delivers LPS from the IM to the OM (Ruiz et al., 2009; Chng et al., 2010a). A sub-complex of the $\beta$-barrel LptD and lipoprotein LptE resides within the OM and accomplishes the final step of inserting LPS into the outer leaflet (Chng et al., 2010b).

A recently described IptE mutation (IptE613) causes defective LPS transport and leads to increased antibiotic sensitivity (Malojčić et al., 2014). To better understand the basis of the lptE613 defect, we isolated spontaneous suppressors that restored antibiotic resistance. One such vancomycin-resistant suppressor mapped to the waaL gene, the product of which is an IM glycosyltransferase that attaches 
eLife digest Tiny Gram-negative bacteria are one of humankind's deadliest foes, causing infections of wounds and the bloodstream that are very hard to treat. Many Gram-negative bacteria are resistant to several common antibiotics, and the few treatments available that can successfully kill the bacteria are often also toxic to the patients. Understanding how these bacteria elude antibiotics could help scientists develop better, less toxic treatments.

Most bacteria are surrounded by a cell wall that helps protect the bacteria and gives them structure. Many broad-spectrum antibiotics, including penicillin and vancomycin, work by interfering with how this protective wall is built from molecules called peptidoglycans. However, Gram-negative bacteria have an outer membrane that prevents many antibiotics from reaching the cell wall, and so the antibiotics are unable to kill the bacteria.

The outer membrane of Gram-negative bacteria is made up of sugars and fatty molecules called lipids. Recently, scientists discovered a mutation that interferes with the movement of the lipid and sugar molecules that make up the outer membrane, which compromises this protective layer and makes the bacteria more susceptible to antibiotics.

To learn more about how this mutation interferes with the defenses of the Gram-negative bacteria Escherichia coli, Grabowicz et al. searched for compensating mutations that can counteract it and restore the antibiotic resistance of these mutant bacteria. The search revealed that a mutation in a gene called waaL increases $E$. coli's resistance to vancomycin, but not to other antibiotics. The gene encodes an enzyme, and the mutant form of the enzyme attaches some peptidoglycans to the surface of the outer membrane instead of incorporating them into the cell wall. The stray peptidoglycans on the cell's surface act as decoys, binding to vancomycin and keeping the drug from reaching its true target-the cell wall. The decoy strategy is similar to a mechanism used by Gram-positive bacteria-which lack a protective outer membrane-to resist vancomycin treatment, which also involves creating sites that bind the drug and keep it from its target.

Vancomycin is not currently used clinically to treat E. coli or other Gram-negative infections because these bacteria are naturally quite resistant for other reasons. However, Grabowicz et al.'s findings do demonstrate how quickly bacteria can adapt and produce new defenses to antibiotics when old strategies fail.

DOI: 10.7554/eLife.05334.002

O-antigen (O-Ag) oligosaccharides to LPS (Han et al., 2012; Ruan et al., 2012). Indeed, the suppressed strain is certainly no more vancomycin sensitive than is the corresponding wild-type control (Figure 1A). However, this suppressor (waaL15 herein) was not specific for IptE613 or even for LPS transport defects. The waaL 15 mutation increases vancomycin resistance in strains carrying bamB or bamE null mutations that disrupt the OM barrier by causing defects in $\beta$-barrel protein assembly (Figure 1B) (Ricci and Silhavy, 2012). Moreover, waaL15 also increases vancomycin-resistance even in the wild-type strain (Figure 1A). The suppressor does not qualitatively improve the OM barrier, since it did not increase resistance against other antibiotics (Figure 1B). So, waaL15 provides a vancomycinspecific resistance mechanism across different strains.

The domesticated E. coli K-12 does not produce the normal substrate (O-Ag) of WaaL (Liu and Reeves, 1994) and a waal deletion does not suppress vancomycin sensitivity, indicating that waaL15 is a gain-of-function mutation. Thus, the WaaL15 mutant O-Ag ligase, which contains an F332S substitution, must have an altered activity. Silver-staining of isolated LPS confirmed that WaaL15 modifies LPS with additional sugars to produce an additional glycoform (LPS*), detected as a higher molecularweight band that is absent in waaL (Figure 2A). WaaL can use two minor saccharide substrates to modify LPS in E. coli K-12: enterobacterial common-antigen (ECA) and colanic acid (CA). ECA-modified LPS is a minor constituent of the OM (Schmidt et al., 1976; Meredith et al., 2007). Production of CA is regulated by the Rcs phospho-relay stress response system, and CA-modified LPS (called 'M-LPS') is only detectable during severe envelope stress (Meredith et al., 2007). Perhaps waaL15 had improved the utility of one, or both, of these substrates. However, LPS silver-staining revealed that LPS* remained detectable when we inactivated biosynthesis of ECA ( $r f f), C A(c p s G)$, or both these polysaccharides (rff cpsG) (Figure 2A). Moreover, if we increase the amounts of a competing substrate by introducing the rcsC137 mutation to activate expression of the genes for CA biosynthesis 
A

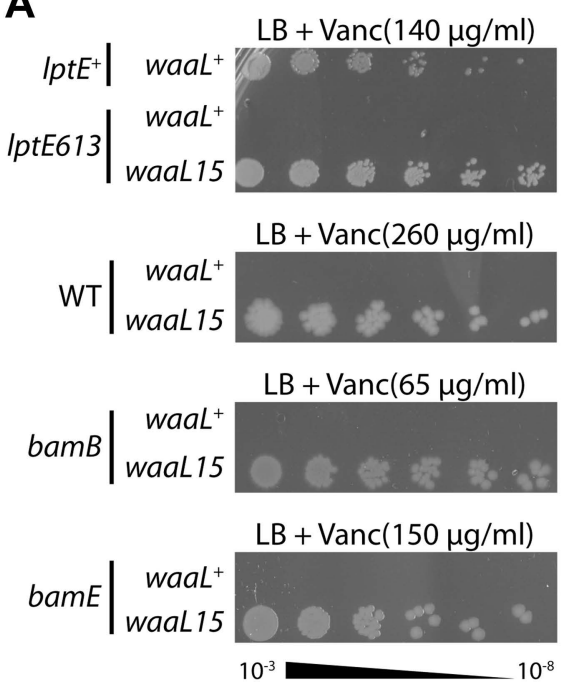

B

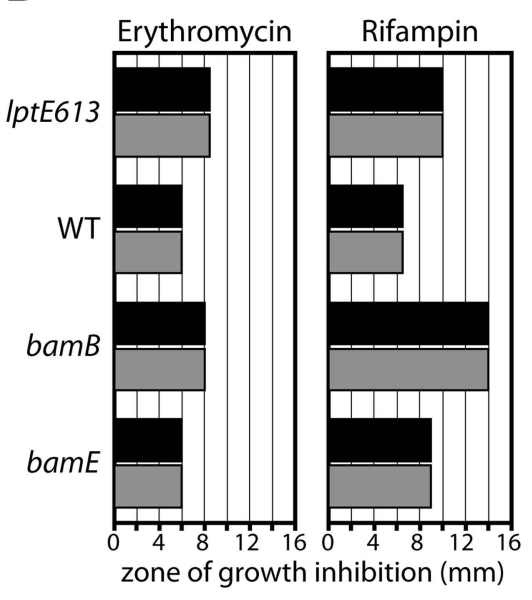

waal $^{+}$

waaL15

Figure 1. A mutant $O$-antigen ligase increases vancomycin resistance. (A) waaL15 provides a strain-independent increase in vancomycin resistance. Isogenic strains, differing by a point mutation in waaL, were plated by serial dilution on LB agar containing indicated amounts of vancomycin. (B) waaL15 does not improve resistance against other antibiotics. Antibiotic discs containing either $15 \mu \mathrm{g}$ erythromycin or $5 \mathrm{\mu g}$ rifampin were placed on LB agar overlays inoculated with the indicated strains. Diametric zones of growth inhibition were measured across the disc. The disc diameter was $6 \mathrm{~mm}$ and this value represents growth at the disc.

DOI: 10.7554/eLife.05334.003

(Gottesman et al., 1985), we actually observed lowered LPS* abundance at the expense of increased M-LPS (Figure 2B). Notably, the decrease in LPS* correlated with a significant reduction in vancomycin-resistance, providing evidence that LPS* molecules directly mediate the resistance (Figure 2C). Similarly, if O-antigen biosynthesis is restored by introducing a wild-type wbbL gene, we observe lowered LPS* at the expense of wild-type LPS and vancomycin resistance is reduced. We conclude that WaaL15 is able to use a new substrate and thereby generate a previously uncharacterized LPS glycoform that provides a specific mechanism for vancomycin resistance.

All native WaaL substrates contain carbohydrates linked to a common undecaprenyl (Und) lipid carrier. PG biosynthesis involves a disaccharide pentapeptide (DPP) linked to the same Und carrier, a molecule called lipid II (Figure 3A). To directly determine if lipid II is a substrate for WaaL15, we treated isolated LPS* with the muralytic enzyme mutanolysin (Figure 3A,B). Digestion of purified LPS*, but not LPS, liberated near-stoichiometric quantities of fragments that were identified by mass spectrometry as DPP or derivatives with a tetrapeptide stem (Figure 3C). Importantly, there is no evidence for cross-linked products suggesting that lipid II was the source of the LPS* glycosylation (Lebar et al., 2013).

There are several carboxypeptidases in the periplasm that remove the terminal D-Alanine (D-Ala) from DPP to produce the tetrapeptide derivative. Indeed, E. coli PG contains negligible amounts of pentapeptide stems (Figure 3-figure supplement 1). Figure $3 \mathrm{C}$ shows that about $50 \%$ of the LPS* is sequestered before it can be attacked by one of these carboxypeptidases. It seemed likely that sequestration happens because the molecule is transported from the periplasm to the cell surface.

Peptide stems from adjacent peptidoglycan strands in the cell wall are cross-linked via transpeptidation between the penultimate D-Ala on one stem and a meso-diaminopimelic acid ( $m$-DAP) residue on a nearby stem (Vollmer et al., 2008). Extensive cross-linking produces a rigid macromolecular meshwork that is vital to cell wall function. Vancomycin binds and sequesters the terminal D-Ala-D-Ala residues of a pentapeptide stem in order to inhibit transpeptidation (Perkins, 1969). Since LPS* was the product of DPP ligation onto LPS, then this modified glycoform should contain vancomycin binding sites. We assessed the ability of purified LPS* to bind vancomycin in vitro. LPS* was immobilized on a carboxymethylated dextran (CM3) chip and we used surface plasmon resonance to monitor 


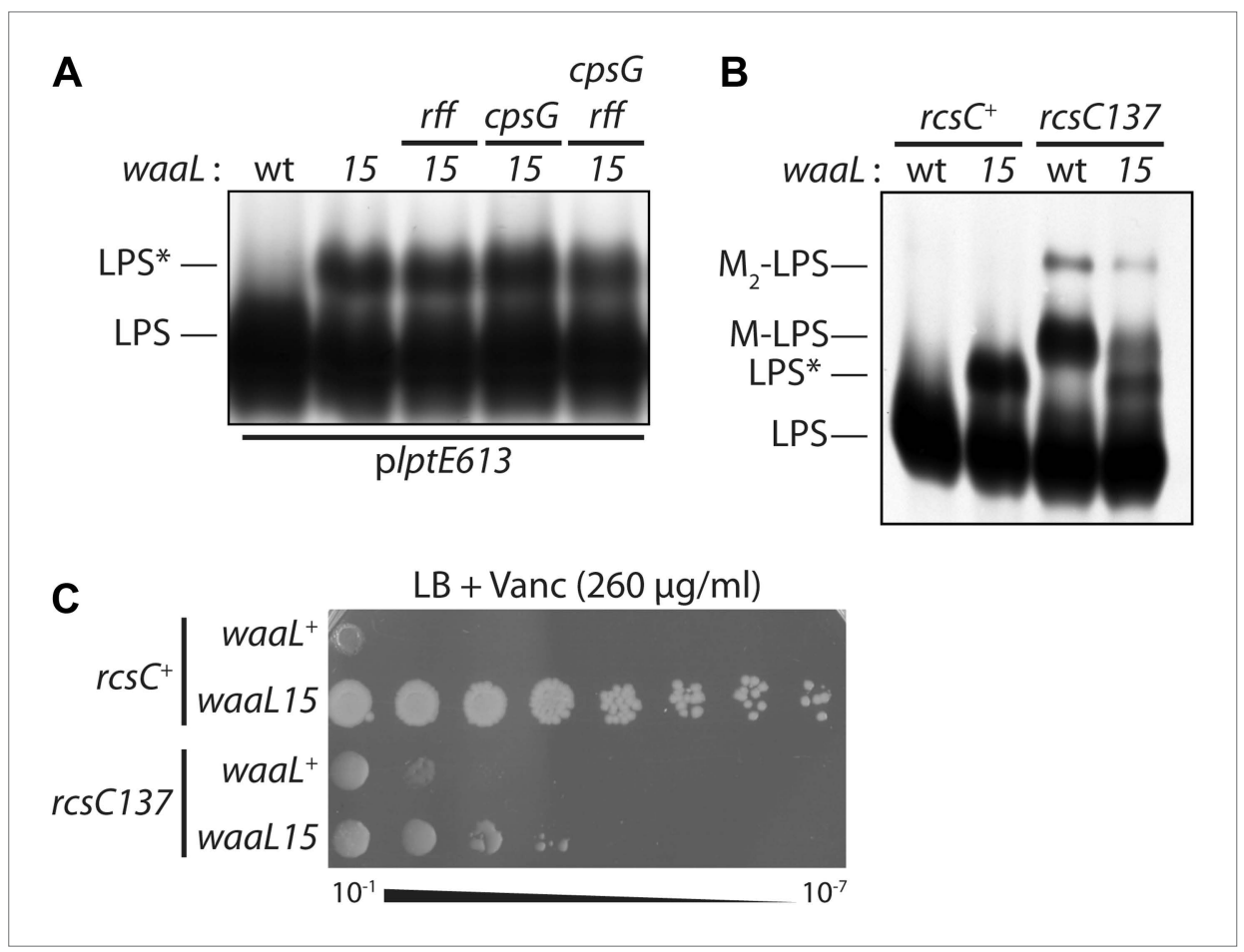

Figure 2. Mutant $\mathrm{O}$-antigen ligase produces a novel form of LPS that is directly responsible for vancomycin resistance. (A) WaaL15 uses a novel substrate to produce a new LPS glycoform. Isolated LPS was resolved by SDS-PAGE and detected by silver staining. A higher molecular weight glycoform (LPS*) appears in waaL15 strains. Mutations that inactivate biosynthesis of ECA (rff::Tn 10-66) or CA ( $\triangle$ cpsG::kan) do not abrogate LPS* production. (B) Overproduction of CA leads to decreased LPS* abundance. Isogenic strains were constructed to express either wt $r c s C^{+}$or the rcs 1137 mutant allele that hyper-activates CA biosynthesis. LPS was isolated and visualized as in (A). LPS molecules modified with a one- or two- CA repeat units are labeled M-LPS and $\mathrm{M}_{2}$-LPS, respectively. (C) Reduced LPS* levels correlate with reduced vancomycin resistance. Strains were plated by serial dilution onto LB agar supplemented with vancomycin.

DOI: 10.7554/eLife.05334.004

interactions with differing concentrations of vancomycin. We were able to measure specific binding of vancomycin to LPS* and to obtain a $K_{d}=0.48 \pm 0.08 \mu \mathrm{M}$ (Figure 4A and Figure 4-figure supplement 1), which is comparable to a reported $K_{d}$ for vancomycin-lipid II interactions in vesicles (Al-Kaddah et al., 2010). Clearly, LPS* molecules include high affinity binding sites for vancomycin.

The ability of LPS* to directly bind vancomycin suggested a possible resistance mechanism, namely that vancomycin is titrated outside the cell. To test this hypothesis, we performed live cell microscopy using a fluorescent vancomycin-BODIPY. We used a wild-type strain background with an intact OM that prevents the influx of vancomycin, to avoid labeling intracellular sites of PG synthesis. Indeed, waaL $L^{+}$cells could not be fluorescently labeled (Figure 4B). On the other hand, we readily detected circumferential labeling of waaL15 bacteria, confirming the presence of accessible D-Ala-D-Ala residues at the cell surface (Figure 4B).

Several vancomycin-resistance mechanisms exist in Gram-positive bacteria, including: alterations in peptidoglycan metabolism can produce thicker cell walls (Cui et al., 2003); and transpeptidation can be reduced to leave more free D-Ala-D-Ala residues within the established cell wall structure (Sieradzki and Tomasz, 1997). It was not immediately apparent to us that any of these strategies could be employed in E. coli since virtually all of the terminal D-Ala residues of DPP are removed either by cross-linking or by the carboxypeptidases. However, we show that the waaL 15 mechanism is comparable since it also increases the number of free D-Ala-D-Ala targets that can tie up vancomycin. Moreover, by displaying D-Ala-D-Ala at the cell surface the waaL15 mutation titrates vancomycin away from the true drug target, in an altogether different cellular compartment. Therefore, LPS* confers resistance by acting as a molecular decoy for vancomycin. Given that Gram-negative bacteria are inherently resistant to vancomycin this decoy mechanism may not be of clinical significance. However, 


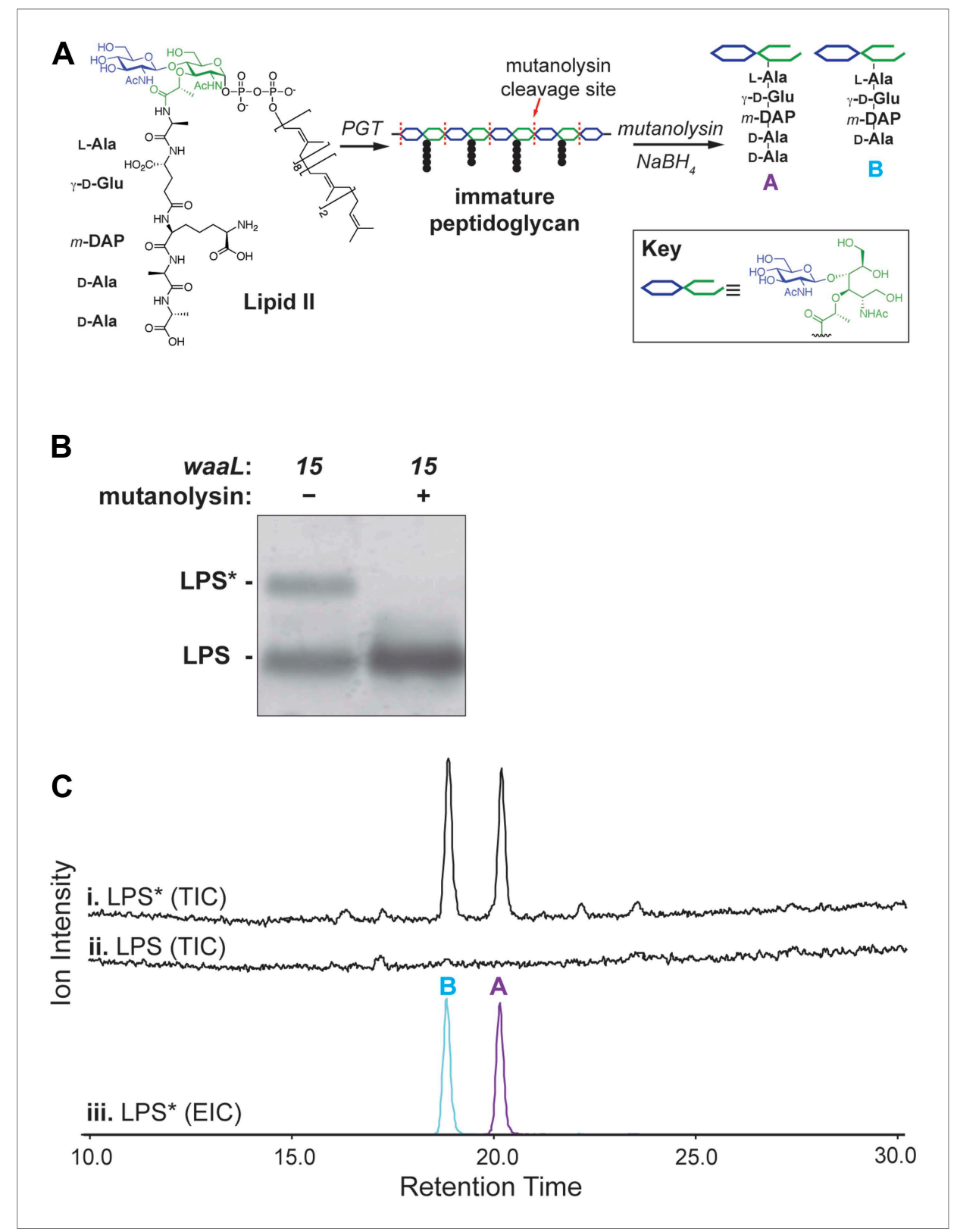

Figure 3. Lipid II is the glycosyl donor for LPS*. (A) Structure of lipid II and schematic of peptidoglycan cleavage by mutanolysin that releases disaccharide pentapeptide ('A') and tetrapeptide ('B') species. (B) Treatment of waaL15 isolated LPS with mutanolysin cleaves the LPS* modification. (C) LPS* is glycosylated with equivalent amounts of lipid II-sourced disaccharide pentapeptide and tetrapeptide. Isolated and purified LPS* from waaL15 and LPS from waa $L^{+}$were treated with mutanolysin and analyzed by LC-MS. Total ion chromatogram for degradation products ( $\mathrm{i}$ and $\mathrm{ii}$ ), and the extracted ion chromatogram for LPS* degradation (iii) are shown. $\mathrm{M}+\mathrm{H}$ and $(\mathrm{M}+2 \mathrm{H}) / 2$ ions corresponding to each fragment were extracted (A: $1013.3+507.2 ; \mathrm{B}: 942.3+471.7$ ). DOI: 10.7554/eLife.05334.005

The following figure supplement is available for figure 3 :

Figure supplement 1 . The waaL15 mutation does not affect the PG cell wall. DOI: 10.7554/eLife.05334.006

the increased resistance it does confer clearly demonstrates the tremendous adaptability of bacteria under antibiotic stress.

The biosynthesis of LPS* is remarkable. Lipid II in E. coli is extremely scarce, its steady-state abundance is thought to be only 1000-2000 molecules per cell (van Heijenoort et al., 1992). Insertion 
A

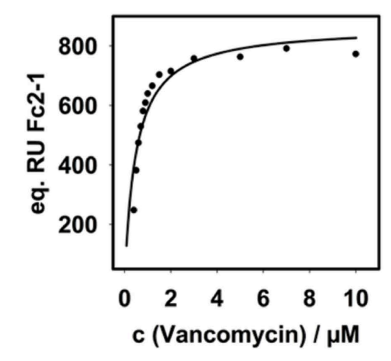

B

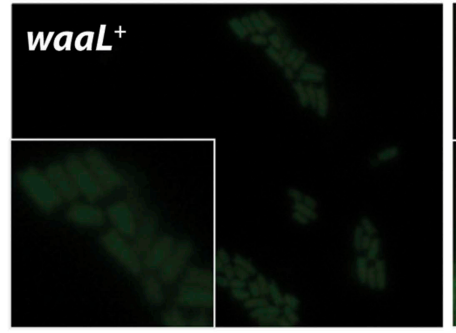

waal 15

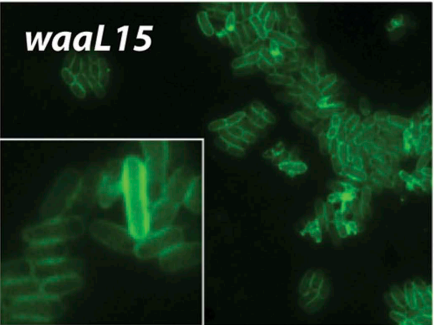

Figure 4. Mutant WaaL attaches peptidoglycan fragments to LPS. (A) LPS* specifically binds vancomycin. Purified LPS $^{*}$ was immobilized on a CM3 chip and varying concentrations of vancomycin were applied. Binding was measured at $25^{\circ} \mathrm{C}$ by surface plasmon resonance. Fitting of equilibrium signal yielded a $K_{d}=0.48 \pm 0.08 \mu \mathrm{M}$. Standard deviation was measured for $0.6 \mu \mathrm{M}$ and $1.2 \mu \mathrm{M}$ and was $\pm 1 \mathrm{RU}$. (B) Vancomycin binds to LPS* at the cell surface. Live exponential-phase growing cells labeled with $1 \mu \mathrm{g} / \mathrm{ml}$ vancomycin-BODIPY for $10 \mathrm{~min}$. Cells were spotted onto M63 minimal medium agar pads and imaged by fluorescence microscopy. DOI: 10.7554/eLife.05334.007

The following figure supplement is available for figure 4:

Figure supplement 1. SPR binding kinetics at $25^{\circ} \mathrm{C}$.

DOI: 10.7554/eLife.05334.008

of new PG is thought to occur via large multiprotein morphogenic complexes: the elongasome and the divisome, responsible for PG synthesis along the lateral cell body and at the septum, respectively. In order to overcome the scarcity of lipid II and limit its diffusion away from sites of PG growth, both complexes are suggested to include at least some of the lipid II biosynthetic enzymes, and the presumed flippases that deliver lipid II from the site of synthesis in the cytoplasm to the site of cell wall assembly in the periplasm (Szwedziak and Löwe, 2013). In this model, the substrate for PG synthesis would be isolated physically from the LPS assembly pathway. LPS is inserted into the OM of each cell at a rate exceeding 70,000 molecules per minute (Lima et al., 2013) and we approximate that one-third of LPS is modified by WaaL15 with lipid II-sourced DPP. Clearly, WaaL15 has ready access to lipid II and this is inconsistent with a model of diffusion-limited lipid II sequestered at the elongasome or divisome complexes. Recent evidence also points to wider lipid II availability (Lee et al., 2014; Sham et al., 2014). Our data indicate that the re-charging of the lipid carrier with new DPP must also be extremely efficient to maintain such a robust pool of PG precursor.

WaaL15 drains the available lipid II pool with no apparent detriment to cell wall integrity (Figure 3figure supplement 1). Lipid II limitation can be revealed by synthetic genetic interactions in a strain lacking the elongasome (Paradis-Bleau et al., 2014), but it is not the recharging of lipid II that is limiting, rather it is the biosynthesis of DPP (Supplementary file 1A).

In many bacteria, LPS is decorated with highly variable O-Ags that are linear polymers of repeating units of 3-6 monosaccharides (Kalynych et al., 2014). In E. coli the multitude of different O-Ags initiate with GlcNAc, ECA also initiates with GlcNAc. In E. coli K-12 when colonic acid is overproduced M-LPS is made from an intitating Glc residue. The F332S mutation broadens substrate specificity of the WaaL glycosyltransferase allowing it to efficiently accept a significantly more bulky initiating MurNAc with an attached oligopeptide stem. The only other glycosyltransferase that is known to use lipid II as a substrate is PgIL from Neisseria and the use required overproduction of the enzyme in E. coli (Faridmoayer et al., 2008). It is also remarkable that we detect no OM biogenesis defect in strains carrying waaL15, demonstrating that the Lpt system is fully competent for the transport and assembly of LPS* despite the addition of both unnatural sugars and peptide stems. Both LPS and PG are pathogen-associated molecular patterns (PAMPs) that potently activate innate immune responses via distinct pathways, and it seems sensible for Gram-negative bacteria to keep these entities separated. We suggest that the F332S substitution has inactivated an exclusion mechanism that prevents WaaL from utilizing the lipid II pool. 


\section{Materials and methods}

\section{Bacterial strains and growth conditions}

Strains and plasmids used in this study are listed in Supplementary file 1B and Supplementary file $1 C$, respectively. Strains were grown in lysogeny broth (LB, $10 \mathrm{~g} / \mathrm{L} \mathrm{NaCl}$ ) or $\mathrm{M} 63$ minimal medium under aeration at $37^{\circ} \mathrm{C}$ unless otherwise noted. When appropriate, media were supplemented with kanamycin (Kan, $25 \mu \mathrm{g} / \mathrm{ml})$, ampicillin (Amp, 25-125 $\mathrm{g} / \mathrm{ml})$, tetracycline (Tet, $20 \mu \mathrm{g} / \mathrm{ml})$, chloramphenicol (Cam, $20 \mu \mathrm{g} / \mathrm{ml}$ ), vancomycin (Vanc, 65-260 $\mu \mathrm{g} / \mathrm{ml}$ ) and arabinose (Ara, 0.2\% vol/vol). Kanamycin deletion-insertion mutations of bamE, cpsG, $\operatorname{mrcA}, \operatorname{mrcB}$, IpoA and IpoB were obtained from the Keio collection (Baba et al., 2006). ECA null rff::Tn10-66 allele was obtained from strain 21566 (MeierDieter et al., 1990). The ompC::Tn5 rcsC137 was obtained from strain SG20803 (Brill et al., 1988). Mutant alleles were introduced by P1 vir transduction.

\section{Isolation and identification of waaL15}

Spontaneous suppressor mutants of strain MG1029 capable of growing on LB plates supplemented with vancomycin $(140 \mu \mathrm{g} / \mathrm{ml})$ were isolated; one such mutant strain was MG1088. The mutation locus conferring vancomycin-resistance in MG1088 was identified by linkage mapping using a library of random mini-Tn10 insertions (Kleckner et alı, 1991). In this way, the tdh::Tn10 allele was found to be approximately $70 \%$ linked to the suppressor mutation waaL15. The F332S mutation was then identified by PCR amplification and sequencing of the waaL locus. The waaL15 mutation was moved into the NR754 wildtype strain by linkage with $t d h:: T n 10$. In order to generate the unmarked waaL15 strain (MG1643) and its wild-type control (MG1642), the tdh::Tn10 mutation was removed from strains MG1210 and MG1211 by first introducing a linked $\Delta$ cysE::kan mutation (Baba et al., 2006), selecting for $K \mathrm{n}^{\mathrm{R}}$ and screening for $\mathrm{Tet}^{\mathrm{S}}$ transductants that were $\operatorname{Vanc}^{\mathrm{R}}$ (waaL15) or $\operatorname{Vanc}^{\mathrm{S}}$ (waaL $L^{+}$). The $\Delta c y s E:$ kan mutation was then replaced with cys $E^{+}$by transduction, selection on $\mathrm{M} 63$ minimal medium, and screening of $\operatorname{Vanc}^{\mathrm{R}} / \mathrm{Vanc}^{\mathrm{S}}$.

\section{Assessment of genetic linkage by co-transduction}

In E. coli, two key PG synthases, PBP1A (mrcA/ponA) and PBP1B (mrcB/ponB), incorporate DPP from Lipid II into PG strands and also mediate transpeptidation (Paradis-Bleau et al., 2010; Typas et al., 2010). Recent evidence suggests that lipid II limitation can be revealed by synthetic genetic interactions in a strain lacking mrcB (Paradis-Bleau et al., 2014). The genetic interaction of PG synthase mutants with waaL15 was assessed as follows. $\mathrm{Kan}^{\mathrm{R}}$-marked null alleles of IpoA, IpoB, mrcA and $\operatorname{mrcB}$ were introduced by P1vir transduction into CAG strains that carry a $\mathrm{Tn} 10$ insertion in a nearby locus (see Supplementary file 1A). $\operatorname{Kan}^{R}$ Tet $^{R}$ transductants were isolated and used to generate P1 vir lysates. These P1 vir were used to transduce waaL+ (MG1642) or waaL15 (MG1643) strains, selecting for the Tn10 marker. The frequency with which the $\mathrm{Kan}^{\mathrm{R}}$-marked Ipo and mrc alleles were co-transduced (genetically linked) was determined by replica plating on LB+Kan. Linkage was assessed in a total of 300 transductants from three independent experiments. A decrease in the cotransduction frequency in waaL15 strains relative to waa $L^{+}$indicates a synthetic interaction between waaL15 and the $\mathrm{Kan}^{\mathrm{R}}$ marked allele. The synthetic interaction between waLL15 and $\mathrm{mrcB} / \mathrm{lpoB}$ null alleles was relieved in strains carrying pMurA when expression of the cloned murA gene (encoding the enzyme responsible for the first committed step in DPP biosynthesis) was induced with $100 \mu \mathrm{M}$ isopropyl $\beta$-D-1thiogalactopyranoside (IPTG). Overexpression of murA increases the cellular pool of UDP-MurNAcpentapeptide and consequently also increases the pool of lipid II.

\section{Analysis of LPS by SDS-PAGE and silver staining}

A total to $1 \times 10^{9}$ cells from liquid culture were taken, pelleted and resuspended $0.05 \mathrm{ml}$ of 'LPS Sample Buffer' $(0.66 \mathrm{M}$ Tris $\mathrm{pH} 7.6,2 \% \mathrm{vol} / \mathrm{vol}$ sodium dodecyl sulfate [SDS], $10 \% \mathrm{vol} / \mathrm{vol}$ glycerol, $4 \% \mathrm{vol} / \mathrm{vol} \beta$-mercaptoethanol, $0.1 \% \mathrm{wt} / \mathrm{vol}$ bromophenol blue). Samples were boiled for $10 \mathrm{~min}$ and allowed to cool to room temperature. $10 \mu \mathrm{l}$ of Proteinase $\mathrm{K}(2.5 \mathrm{mg} / \mathrm{ml}$, in LPS Sample Buffer) was added and samples were incubated at $56^{\circ} \mathrm{C}$ for $16 \mathrm{hr}$. LPS samples were then resolved by SDS-PAGE and silver stained as described previously (Tsai and Frasch, 1981). By quantifying band density using ImageJ, we measured that LPS* constitutes $29 \pm 1 \%$ of the total LPS in waaL15 samples.

\section{Antibiotic disc diffusion assay}

$3 \mathrm{ml}$ of molten LB Top agar ( $0.75 \%$ agar) was inoculated with $0.1 \mathrm{ml}$ of overnight culture. The mixture was poured onto a LB agar plate (1.5\% agar, ) and allowed to set. Antibiotic discs (BD Sensi-Disc) were 
placed on the Top agar overlay and plates were incubated overnight at $37^{\circ} \mathrm{C}$. The 'zone of growth inhibition' was measured across the antibiotic disc.

\section{Fluorescence microscopy}

Overnight cultures were sub-cultured at 1:100 into fresh LB broth and grown for $1.5 \mathrm{hr}$. A $1 \mathrm{ml}$ aliquot was taken, pelleted and was twice washed with $1 \mathrm{ml} \mathrm{M63}$ medium. Cells were resuspended in $0.1 \mathrm{ml}$ of M63 broth containing $1 \mu \mathrm{g} / \mathrm{ml}$ of vancomycin-BODIPY-FL (Life Technologies, V-34850). Cells were incubated at room temperature for $10 \mathrm{~min}$ and then washed twice with $1 \mathrm{ml} \mathrm{M63} \mathrm{broth}$. Cells were then resuspended in $0.03 \mathrm{ml}$ of M63 broth, and approximately $2 \mathrm{ml}$ was spotted onto an M63-agarose pad. Cells were immediately visualized on a Nikon Eclipse 90i microscope with a Nikon Plan Apo 1.4/100 × Oil Ph3 phase objective.

\section{LPS Purification}

E. coli MG1210 and MG1211 were each grown in $4 \times 1.5$ I LB medium shaking at $37^{\circ} \mathrm{C}$ overnight to stationary phase. The cells were harvested by centrifugation for $15 \mathrm{~min}$ at $5000 \times \mathrm{g}, 4^{\circ} \mathrm{C}$ and washed with water $(700 \mathrm{ml})$ and ethanol $(40 \mathrm{ml})$ once, then twice with acetone $(40 \mathrm{ml})$. After drying the cell pellet in a desiccator over night in vacuo, PCP (Phenol-Chloroform-petroleum ether) method was used for rough LPS extraction (Galanos et al., 1969).

\section{PG Purification}

E. coli MG1210 and MG1211 were each grown in $500 \mathrm{ml} \mathrm{LB}$ medium shaking at $37^{\circ} \mathrm{C}$ to stationary phase ( $6 \mathrm{hr}$ ). The cell wall was isolated from the culture as described by Glauner et al. (1988) and Uehara et al. (2009), with modifications described below. The cells were resuspended in $20 \mathrm{ml}$ phosphate buffered saline (PB, pH $=7.4$ ) and boiled for $30 \mathrm{~min}$ in $80 \mathrm{ml} 5 \% \mathrm{SDS}$. After the samples cooled, they were pelleted $\left(14,000 \mathrm{rpm}, 25^{\circ} \mathrm{C}, 1 \mathrm{hr}\right)$ and washed six times by pelleting $\left(14,000 \mathrm{rpm}, 25^{\circ} \mathrm{C}, 1 \mathrm{hr}\right)$ from $50 \mathrm{ml}$ water aliquots to remove the SDS. The samples were resuspended in $1 \mathrm{ml} \mathrm{PBS,} \mathrm{treated} \mathrm{with}$ $\alpha$-amidase $\left(100 \mu l, 2 \mathrm{mg} / \mathrm{ml}\right.$ stock in $50 \%$ glycerol, Sigma A-6380) and incubated at $37^{\circ} \mathrm{C}$ with shaking for $2 \mathrm{hr}$. To cleave proteins attached to the cell wall, a-chymotrypsin ( $100 \mu \mathrm{l}, 3 \mathrm{mg} / \mathrm{ml}$ in $50 \%$ glycerol, Sigma C3142) was added, and the samples were incubated at $37^{\circ} \mathrm{C}$ with shaking overnight. An additional aliquot of $\alpha$-chymotrypsin $(100 \mu \mathrm{l})$ was added, and the samples were digested for an additional $4 \mathrm{hr}$. To remove the proteins, SDS was added to a final concentration of $1 \%$, and the samples were incubated at $95^{\circ} \mathrm{C}$ for $1 \mathrm{hr}$. After cooling, the samples were again pelleted $\left(14,000 \mathrm{rpm}, 25^{\circ} \mathrm{C}, 1 \mathrm{hr}\right)$ and washed with water repeatedly $(4 \times 25 \mathrm{ml})$ to remove the SDS. The final peptidoglycan (PG) samples were resuspended in $500 \mu \mathrm{l} 0.02 \%$ azide and stored at $4^{\circ} \mathrm{C}$.

\section{Mutanolysin digestion and analysis}

The PG composition was analyzed by LC/MS as previously described (Lebar et al., 2013). The method was also used to analyze LPS samples. The glycosylhydrolase mutanolysin liberated DPP and disaccharide tetrapeptide from LPS*. Aliquots (40 $\mu \mathrm{l}$ ) of PG (from MG1210 and MG1211) and LPS (from MG1210 and MG1211) were incubated with mutanolysin (10 U, $2.5 \mu \mathrm{l}, 4000 \mathrm{U} / \mathrm{ml}$, Sigma M9901, stored at $-20^{\circ} \mathrm{C}$ in $50 \mathrm{mM} \mathrm{TES}, \mathrm{pH} 7.0,1 \mathrm{mM} \mathrm{MgCl}, 10 \%$ glycerol) in $50 \mathrm{mM}$ sodium phosphate buffer $\left(\mathrm{pH} 6.0,100 \mu \mathrm{l}\right.$ total volume) at $37^{\circ} \mathrm{C}$ with shaking overnight. Another aliquot of mutanolysin $(10 \mathrm{U}$, $2.5 \mu$ l) was added, and the mixture was incubated at $37^{\circ} \mathrm{C}$ with shaking for $3 \mathrm{hr}$. Insoluble particles were separated by centrifugation $(16,000 \times \mathrm{g})$. The supernatant, containing soluble fragments, was treated with sodium borohydride $(10 \mathrm{mg} / \mathrm{ml}$ in water, $100 \mu \mathrm{l})$ at room temperature for $30 \mathrm{~min}$. Phosphoric acid $(20 \%, 12 \mu \mathrm{l})$ was then added to adjust $\mathrm{pH}$ to $\sim 4$. When bubbling ceased, the samples were lyophilized and re-dissolved in $25 \mu \mathrm{l}$ water, which was analyzed on LC/MS. LC/MS analysis was conducted with ESI-MS operating in positive mode. The instrument was equipped with a Waters Symmetry Shield RP18 column $(5 \mu \mathrm{m}, 3.9 \times 150 \mathrm{~mm})$ with matching column guard. The fragments were separated using the following method: $0.5 \mathrm{ml} / \mathrm{min} \mathrm{H}_{2} \mathrm{O}(0.1 \%$ formic acid) for $5 \mathrm{~min}$ followed by a gradient of $0 \% \mathrm{ACN}\left(0.1 \%\right.$ formic acid) $/ \mathrm{H}_{2} \mathrm{O}(0.1 \%$ formic acid) to $20 \% \mathrm{ACN}(0.1 \%$ formic acid $) /$ $\mathrm{H}_{2} \mathrm{O}(0.1 \%$ formic acid) over $40 \mathrm{~min}$.

\section{Surface plasmon resonance analysis}

Purified LPS $(0.5 \mathrm{mg} / \mathrm{ml})$ from strains MG1210 or MG1211 were extruded in $20 \mathrm{mM}$ Tris/ $\mathrm{HCl} \mathrm{pH} 8$, $150 \mathrm{mM} \mathrm{NaCl}$ and immobilized on poly-L-lysine coated CM3 Biacore chips on the active and reference channel, respectively (Malojčić et al., 2014). All experiments were performed using a Biacore X100 
instrument at $25^{\circ} \mathrm{C}$ at a flow rate of $10 \mu \mathrm{l} / \mathrm{min}$ with $20 \mathrm{mM}$ Tris/ $\mathrm{HCl} \mathrm{pH} 8,150 \mathrm{mM} \mathrm{NaCl}$ buffer. Different concentrations of vancomycin were injected for $400 \mathrm{~s}$ and dissociation was recorded for another $500 \mathrm{~s}$ to return to baseline. No binding was observed to the reference channel. The equilibrium signal in the difference channel was fitted to $f=\operatorname{Bmax}{ }^{*} a b s(x) /(K d+a b s(x))$ with $R^{2}=0.88$. Standard deviation was measured for $0.6 \mu \mathrm{M}$ and $1.2 \mu \mathrm{M}$ vancomycin in triplicate and did not exceed $1 \mathrm{RU}$.

\section{Acknowledgements}

We thank Natividad Ruiz, Kerrie L May, Robert S Dwyer, and members of the Silhavy lab for insights, comments and suggestions.

\section{Additional information}

Funding

\begin{tabular}{|c|c|c|}
\hline Funder & Grant reference number & Author \\
\hline National Institutes of Health & GM034821 & $\begin{array}{l}\text { Marcin Grabowicz, } \\
\text { Thomas J Silhavy }\end{array}$ \\
\hline National Institutes of Health & GM066174 & $\begin{array}{l}\text { Dorothee Andres, } \\
\text { Matthew D Lebar, } \\
\text { Daniel Kahne }\end{array}$ \\
\hline Swiss National Science Foundation & P300P2_147905 & Goran Malojčić \\
\hline National Institutes of Health & GM103056 & Matthew D Lebar \\
\hline Swiss National Science Foundation & PA00P3_134194 & Goran Malojčić \\
\hline
\end{tabular}

The funders had no role in study design, data collection and interpretation, or the decision to submit the work for publication.

Author contributions

MG, DA, MDL, Conception and design, Acquisition of data, Analysis and interpretation of data, Drafting or revising the article; GM, Conception and design; DK, TJS, Conception and design, Analysis and interpretation of data, Drafting or revising the article

\section{Additional files}

Supplementary file

- Supplementary file 1. (A) Synthetic interactions between waaL15 and mutations affecting the elongasome due to limited lipid II availability. (B) Strains used in this study. (C) Plasmids used in this study.

DOI: 10.7554/eLife.05334.009

\section{References}

Al-Kaddah S, Reder-Christ K, Klocek G, Wiedemann I, Brunschweiger M, Bendas G. 2010. Analysis of membrane interactions of antibiotic peptides using ITC and biosensor measurements. Biophysical Chemistry 152:145-152. doi: 10.1016/j.bpc.2010.09.002.

Baba T, Ara T, Hasegawa M, Takai Y, Okumura Y, Baba M, Datsenko KA, Tomita M, Wanner BL, Mori H. 2006. Construction of Escherichia coli K-12 in-frame, single-gene knockout mutants: the Keio collection. Molecular Systems Biology 2:2006.0008. doi: 10.1038/msb4100050.

Brill JA, Quinlan-Walshe C, Gottesman S. 1988. Fine-structure mapping and identification of two regulators of capsule synthesis in Escherichia coli K-12. Journal of Bacteriology 170:2599-2611.

Chng SS, Gronenberg LS, Kahne D. 2010a. Proteins required for lipopolysaccharide assembly in Escherichia coli form a transenvelope complex. Biochemistry 49:4565-4567. doi: 10.1021/bi100493e.

Chng SS, Ruiz N, Chimalakonda G, Silhavy TJ, Kahne D. 2010b. Characterization of the two-protein complex in Escherichia coli responsible for lipopolysaccharide assembly at the outer membrane. Proceedings of the National Academy of Sciences of USA 107:5363-5368. doi: 10.1073/pnas.0912872107.

Cui L, Ma XX, Sato K, Okuma K, Tenover FC, Mamizuka EM, Gemmell CG, Kim MN, Ploy MC, El-Solh N, Ferraz V, Hiramatsu K. 2003. Cell wall thickening is a common feature of vancomycin resistance in Staphylococcus aureus. Journal of Clinical Microbiology 41:5-14. doi: 10.1128/JCM.41.1.5-14.2003. 
Eggert US, Ruiz N, Falcone BV, Branstrom AA, Goldman RC, Silhavy TJ, Kahne D. 2001. Genetic basis for activity differences between vancomycin and glycolipid derivatives of vancomycin. Science 294:361-364. doi: 10.1126/ science.1063611.

Faridmoayer A, Fentabil MA, Haurat MF, Yi W, Woodward R, Wang PG, Feldman MF. 2008. Extreme substrate promiscuity of the Neisseria oligosaccharyl transferase involved in protein O-glycosylation. The Journal of Biological Chemistry 283:34596-34604. doi: 10.1074/jbc.M807113200.

Galanos C, Lüderitz O, Westphal O. 1969. A new method for the extraction of R lipopolysaccharides. European Journal of Biochemistry/FEBS 9:245-249. doi: 10.1111/j.1432-1033.1969.tb00601.x.

Glauner B, Höltje JV, Schwarz U. 1988. The composition of the murein of Escherichia coli. The Journal of Biological Chemistry 263:10088-10095.

Gottesman S, Trisler P, Torres-Cabassa A. 1985. Regulation of capsular polysaccharide synthesis in Escherichia coli K-12: characterization of three regulatory genes. Journal of Bacteriology 162:1111-1119.

Han W, Wu B, Li L, Zhao G, Woodward R, Pettit N, Cai L, Thon V, Wang PG. 2012. Defining function of lipopolysaccharide O-antigen ligase WaaL using chemoenzymatically synthesized substrates. The Journal of Biological Chemistry 287:5357-5365. doi: 10.1074/jbc.M111.308486.

Kalynych S, Morona R, Cygler M. 2014. Progress in understanding the assembly process of bacterial O-antigen. FEMS Microbiology Reviews 38:1048-1065. doi: 10.1111/1574-6976.12070.

Kamio Y, Nikaido H. 1976. Outer membrane of Salmonella Typhimurium: accessibility of phospholipid head groups to phospholipase $\mathrm{C}$ and cyanogen bromide activated dextran in the external medium. Biochemistry 15:2561-2570. doi: 10.1021/bi00657a012.

Kleckner N, Bender J, Gottesman S. 1991. Uses of transposons with emphasis on Tn10. Methods in Enzymology 204:139-180. doi: 10.1016/0076-6879(91)04009-D.

Lebar MD, Lupoli TJ, Tsukamoto H, May JM, Walker S, Kahne D. 2013. Forming cross-linked peptidoglycan from synthetic Gram-negative Lipid II. Journal of the American Chemical Society 135:4632-4635. doi: 10.1021/ ja312510m.

Lee TK, Tropini C, Hsin J, Desmarais SM, Ursell TS, Gong E, Gitai Z, Monds RD, Huang KC. 2014. A dynamically assembled cell wall synthesis machinery buffers cell growth. Proceedings of the National Academy of Sciences of USA 111:4554-4559. doi: 10.1073/pnas.1313826111.

Lima S, Guo MS, Chaba R, Gross CA, Sauer RT. 2013. Dual molecular signals mediate the bacterial response to outer-membrane stress. Science 340:837-841. doi: 10.1126/science.1235358.

Liu D, Reeves P. 1994. Escherichia coli K12 regains its O antigen. Nature Reviews. Microbiology 140:49-57. doi: 10.1099/13500872-140-1-49.

Malojčić G, Andres D, Grabowicz M, George AH, Ruiz N, Silhavy TJ, Kahne D. 2014. LptE binds to and alters the physical state of LPS to catalyze its assembly at the cell surface. Proceedings of the National Academy of Sciences of USA 111:9467-9472. doi: 10.1073/pnas.1402746111.

Meier-Dieter U, Starman R, Barr K, Mayer H, Rick PD. 1990. Biosynthesis of enterobacterial common antigen in Escherichia coli. Biochemical characterization of Tn10 insertion mutants defective in enterobacterial common antigen synthesis. The Journal of Biological Chemistry 265:13490-13497.

Meredith TC, Mamat U, Kaczynski Z, Lindner B, Holst O, Woodard RW. 2007. Modification of lipopolysaccharide with colanic acid (M-antigen) repeats in Escherichia coli. The Journal of Biological Chemistry 282:7790-7798. doi: 10.1074/jbc.M611034200.

Nikaido H. 2003. Molecular basis of bacterial outer membrane permeability revisited. Microbiology and Molecular Biology Reviews 67:593-656. doi: 10.1128/MMBR.67.4.593-656.2003.

Osborn MJ, Gander JE, Parisi E, Carson J. 1972. Mechanism of assembly of the outer membrane of Salmonella Typhimurium. Isolation and characterization of cytoplasmic and outer membrane. The Journal of Biological Chemistry 247:3962-3972.

Paradis-Bleau C, Kritikos G, Orlova K, Typas A, Bernhardt TG. 2014. A genome-wide screen for bacterial envelope biogenesis mutants identifies a novel factor involved in cell wall precursor metabolism. PLOS Genetics 10:e1004056. doi: 10.1371/journal.pgen.1004056.

Paradis-Bleau C, Markovski M, Uehara T, Lupoli TJ, Walker S, Kahne D, Bernhardt TG. 2010. Lipoprotein cofactors located in the outer membrane activate bacterial cell wall polymerases. Cell 143:1110-1120. doi: 10.1016/j.cell.2010.11.037.

Perkins HR. 1969. Specificity of combination between mucopeptide precursors and vancomycin or ristocetin. The Biochemical Journal 111:195-205.

Raetz CR, Whitfield C. 2002. Lipopolysaccharide endotoxins. Annual Review of Biochemistry 71:635-700. doi: 10.1146/annurev.biochem.71.110601.135414.

Ricci DP, Silhavy TJ. 2012. The Bam machine: a molecular cooper. Biochimica et Biophysica Acta 1818: 1067-1084. doi: 10.1016/j.bbamem.2011.08.020.

Ruan X, Loyola DE, Marolda CL, Perez-Donoso JM, Valvano MA. 2012. The WaaL O-antigen lipopolysaccharide ligase has features in common with metal ion-independent inverting glycosyltransferases. Glycobiology 22:288-299. doi: 10.1093/glycob/cwr150.

Ruiz N, Falcone B, Kahne D, Silhavy TJ. 2005. Chemical conditionality: a genetic strategy to probe organelle assembly. Cell 121:307-317. doi: 10.1016/j.cell.2005.02.014.

Ruiz N, Kahne D, Silhavy TJ. 2009. Transport of lipopolysaccharide across the cell envelope: the long road of discovery. Nature Reviews. Microbiology 7:677-683. doi: 10.1038/nrmicro2184.

Schmidt G, Mannel D, Mayer H, Whang HY, Neter E. 1976. Role of a lipopolysaccharide gene for immunogenicity of the enterobacterial common antigen. Journal of Bacteriology 126:579-586. 
Sham LT, Butler EK, Lebar MD, Kahne D, Bernhardt TG, Ruiz N. 2014. MurJ is the flippase of lipid-linked precursors for peptidoglycan biogenesis. Science 345:220-222. doi: 10.1126/science.1254522.

Sieradzki K, Tomasz A. 1997. Inhibition of cell wall turnover and autolysis by vancomycin in a highly vancomycinresistant mutant of Staphylococcus aureus. Journal of Bacteriology 179:2557-2566.

Singer M, Baker TA, Schnitzler G, Deischel SM, Goel M, Dove W, Jaacks KJ, Grossman AD, Erickson JW, Gross CA. 1989. A collection of strains containing genetically linked alternating antibiotic resistance elements for genetic mapping of Escherichia coli. Microbiological Reviews 53:1-24.

Szwedziak P, Löwe J. 2013. Do the divisome and elongasome share a common evolutionary past? Current Opinion in Microbiology 16:745-751. doi: 10.1016/j.mib.2013.09.003.

Tsai CM, Frasch CE. 1981. A sensitive silver stain for detecting lipopolysaccharides in polyacrylamide gels. Analytical Biochemistry 119:115-119. doi: 10.1016/0003-2697(82)90673-X.

Typas A, Banzhaf M, van den Berg van Saparoea B, Verheul J, Biboy J, Nichols RJ, Zietek M, Beilharz K, Kannenberg K, von Rechenberg M, Breukink E, den Blaauwen T, Gross CA, Vollmer W. 2010. Regulation of peptidoglycan synthesis by outer-membrane proteins. Cell 143:1097-1109. doi: 10.1016/j.cell.2010.11.038.

Uehara T, Dinh T, Bernhardt TG. 2009. LytM-domain factors are required for daughter cell separation and rapid ampicillin-induced lysis in Escherichia coli. Journal of Bacteriology 191:5094-5107. doi: 10.1128/JB.00505-09.

van Heijenoort Y, Gómez M, Derrien M, Ayala J, van Heijenoort J. 1992. Membrane intermediates in the peptidoglycan metabolism of Escherichia coli: possible roles of PBP $1 \mathrm{~b}$ and PBP 3. Journal of Bacteriology 174:3549-3557.

Vollmer W, Blanot D, de Pedro MA. 2008. Peptidoglycan structure and architecture. FEMS Microbiology Reviews 32:149-167. doi: 10.1111/j.1574-6976.2007.00094.x.

Zhou Z, White KA, Polissi A, Georgopoulos C, Raetz CR. 1998. Function of Escherichia coli MsbA, an essential $A B C$ family transporter, in lipid A and phospholipid biosynthesis. The Journal of Biological Chemistry 273:12466-12475. doi: 10.1074/jbc.273.20.12466. 